\title{
Threshold shift and above-threshold multiphoton ionization of atomic hydrogen in intense laser fields
}

\author{
Shih-I Chu* and J. Cooper \\ Joint Institute for Laboratory Astrophysics, University of Colorado and National Bureau of Standards, \\ Boulder, Colorado 80309 \\ (Received 21 May 1985)
}

\begin{abstract}
Accurate $a b$ initio nonperturbative $L^{2}$ non-Hermitian Floquet calculations for intensitydependent threshold shifts and ground-state total ionization widths (rates) for one-, two-, and threephoton-dominant intense-field ionization of atomic hydrogen are presented. The results show the importance of both the ac Stark shift and the pondermotive potential in the determination of the net threshold shift. In addition, branching ratios to individual continua have been estimated, yielding physical insights regarding the general features and mechanisms of the frequency- and intensitydependent continuum-continuum transitions and "peak switching" phenomena in the abovethreshold ionization processes.
\end{abstract}

\section{INTRODUCTION}

Several recent experiments with multiphoton ionization (MPI) of atoms in strong laser fields reveal interesting and unexpected structures in the energy spectra of the ejected electrons. ${ }^{1-3}$ When the atoms are irradiated by a weak electromagnetic field of frequency $\omega$, the energy of the emitted electron is equal to $N_{m} \hbar \omega-E_{0}$, where $E_{0}$ is the ionization energy of the electron, and $N_{m}$ is the minimum number of photons required to ionize the atom, i.e., $\left(N_{m}-1\right) \hbar \omega<E_{0}<N_{m} \hbar \omega$. At strong fields, additional photons may be absorbed and the electron energy spectrum consists of several peaks at energies $\left(N_{m}+S\right) \hbar \omega-E_{0}$ with $S=0,1,2, \ldots$. This phenomenon has been called "above-threshold ionization" (ATI). At still higher fields, the electron peaks broaden and shift, and the slowest electron peak eventually disappears. Various theoretical models have been proposed to account for the observations. These include the pondermotive force and the shift of the ionization potential ${ }^{4,5}$ and continuum-continuum transitions, ${ }^{6-10}$ etc. A qualitative unified interpretation of ATI electron spectra has been given by Szöke. ${ }^{11} A b$ initio nonperturbative calculations for intense-field MPI and ATI processes are rare, if there are any, due to the complexity of the problem.

In this paper we report accurate nonperturbative calculations of intensity- and frequency-dependent ac Stark shifts, total MPI widths (rates), and ionization potentials of atomic hydrogen subjected to high-power linearly polarized monochromatic laser fields $\left(I \sim 10^{13}-10^{15}\right.$ $\mathrm{W} / \mathrm{cm}^{2}$ ). We also report approximate estimates of the branching ratios in ATI electron spectra. Experimental investigation of MPI of the $\mathrm{H}$ atom has become feasible only very recently, ${ }^{12}$ and the corresponding ATI experiment is planned. The shift and width presented here are two of the key parameters for experimental MPI and ATI analysis. The branching-ratio estimate also leads to approximate prediction of the frequency and intensity range at which one is likely to see the ATI phenomenon and continuum-continuum transitions in atomic hydrogen.

\section{METHOD}

The method we adopt here is based on the extension of the $L^{2}$ non-Hermitian Floquet formulation of the multiphoton process developed by $\mathrm{Chu}$ and Reinhardt. ${ }^{13}$ The theory allows nonperturbative and self-consistent treatment of intense field effects (in that all atomic levels are simultaneously shifted and broadened by the periodic external field), and straightforward inclusion of free-free transitions and the effects of coupling between electronic continua.

The time-dependent Hamiltonian for the system under consideration is, in atomic units,

$$
\hat{H}(t)=-\frac{1}{2} \nabla^{2}-\frac{1}{r}-F z \cos (\omega t)
$$

which describes the interaction of atomic hydrogen with a monochromatic, linearly polarized, coherent field of frequency $\omega$ and peak field strength $F . \hat{H}(t)$ can be transformed into an equivalent time-independent Floquet Hamiltonian $\hat{H}_{F}$ by extending Shirley's finite-level theory $^{14}$ to infinite level. ${ }^{13}$ Further, the dilation transformation, ${ }^{15} r \rightarrow r e^{i \alpha}$, is applied to the Floquet Hamiltonian,

$$
\hat{H}_{F}(\mathbf{r}) \rightarrow \hat{H}_{F}\left(\mathbf{r} e^{i \alpha}\right) \equiv \hat{H}_{F}(\alpha)
$$

$\hat{H}_{F}(\alpha)$ has isolated eigenvalues in the lower halfcomplex-plane which correspond to decaying complex quasienergy states (QES) or dressed states. The dilation transformation distorts the continuous spectrum away from the real axis, exposing the complex QES in appropriate higher Riemann sheets, and also allowing use of biorthogonal variational expansions employing $L^{2}$ basis functions chosen from a complete discrete basis. The use of a complete $L^{2}$ basis obviates the necessity for explicit introduction of exact atomic bound and continuum states, thus reducing all computations to those involving finitedimensional non-Hermitian Floquet matrices. The use of complex coordinates not only allows direct calculation of eigenvalue parameters associated with complex QES, but 
completely avoids numerical problems arising from strong coupling between overlapping atomic continua. The real parts of the complex eigenvalues of $\hat{H}_{F}(\alpha)$ provide the ac Stark shifts, whereas the imaginary parts determine directly the total MPI widths (rates). We note in passing that the $L^{2}$ non-Hermitian Floquet theory has been recently generalized to the nonperturbative treatment of multiphoton dissociation of molecules. ${ }^{16}$

The structure of the Floquet Hamiltonian $\hat{H}_{F}(\alpha)$ for linearly polarized fields is given in Fig. 1. This form of the matrix is the same as used earlier by Chu and Reinhardt. ${ }^{13}$ Generalization to circular polarization has been given in Ref. 17. Figure 1 shows that $\hat{H}_{F}(\alpha)$ is a complex symmetric matrix and is composed of Floquet blocks, of type $A$, which are in turn composed of angular momentum blocks $\widetilde{S}, \widetilde{P}, \widetilde{D}, \ldots$; both types of blocks are coupled by the dipole coupling elements $V_{l, l \pm 1}$. The atomic blocks $\widetilde{S}, \widetilde{P}, \widetilde{D}, \ldots$ are made discrete by use of a finite subset of the complete Laguerre basis $r^{l+1} e^{-\lambda r / 2} L_{n}^{2 l+2}(\lambda r), n=0,1,2, \ldots$ which gives a Pollaczek quadrature ${ }^{18}$ representation of the bound and continuum contributions to the spectral resolution of the hydrogenic Hamiltonian. The convergence of the MPI calculation must be obtained with respect to the number of Floquet blocks, as well as with respect to the basis size and number of angular momentum blocks. ${ }^{13}$ To the extent that enough Floquet blocks are included to obtain convergence, a completely nonperturbative result is obtained in that all orders of perturbation theory are included, and all relevant processes involving differing photon numbers are simultaneously and self-consistently included.
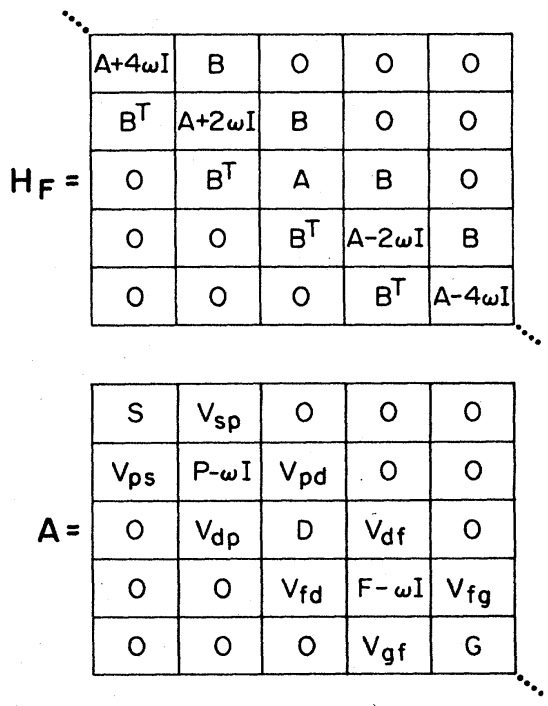

$B=$\begin{tabular}{|c|c|c|c|c|}
\hline 0 & 0 & 0 & 0 & 0 \\
\hline$v_{p s}$ & 0 & $v_{p d}$ & 0 & 0 \\
\hline 0 & 0 & 0 & 0 & 0 \\
\hline 0 & 0 & $v_{f d}$ & 0 & $v_{f g}$ \\
\hline 0 & 0 & 0 & 0 & 0 \\
\hline
\end{tabular}

FIG. 1. Structure of the Floquet Hamiltonian for atomic MPI.

TABLE I. Intensity-dependent complex quasienergy $\left(E_{R},-i \Gamma / 2\right)$ in atomic units of the perturbed ground state of the $\mathrm{H}$ atom nearby the $N_{m}=1$ region $\left(\omega \sim 0.5\right.$ a.u.). The rms field strength of $F_{\mathrm{rms}}=1.0$ a.u. corresponds to an $\mathrm{rms}$ intensity of $7.0 \times 10^{16} \mathrm{~W} / \mathrm{cm}^{2}$.

\begin{tabular}{|c|c|c|c|c|c|}
\hline$\omega$ & $E_{R}$ & $-\Gamma / 2$ & $\omega$ & $E_{R}$ & $-\Gamma / 2$ \\
\hline \multicolumn{3}{|c|}{$F_{\mathrm{rms}}=0.01^{\mathrm{a}}$ a.u. } & \multicolumn{3}{|c|}{$F_{\mathrm{rms}}=0.05^{\mathrm{a}}$ a.u. } \\
\hline 0.60 & -0.4998351 & $-0.1253(-3)^{b}$ & 0.60 & -0.495844 & $-0.3144(-2)$ \\
\hline 0.55 & -0.4998104 & $-0.1729(-3)$ & 0.55 & -0.495201 & $-0.4355(-2)$ \\
\hline 0.50 & -0.4997843 & $-0.2456(-3)$ & 0.50 & -0.494544 & $-0.6244(-2)$ \\
\hline 0.495 & -0.4997818 & $-0.2551(-3)$ & 0.495 & -0.494514 & $-0.6414(-2)$ \\
\hline \multirow[t]{2}{*}{0.490} & -0.4997804 & $-0.2647(-3)$ & 0.490 & -0.494380 & $-0.6510(-2)$ \\
\hline & \multicolumn{2}{|c|}{$F_{\mathrm{rms}}=0.025^{\mathrm{a}}$ a.u. } & \multicolumn{3}{|c|}{$F_{\mathrm{rms}}=0.075^{\mathrm{c}}$ a.u. } \\
\hline 0.60 & -0.498967 & $-0.7836(-3)$ & 0.70 & -0.49293 & $-0.3966(-2)$ \\
\hline 0.55 & -0.498812 & $-0.1083(-2)$ & 0.60 & -0.49055 & $-0.7107(-2)$ \\
\hline 0.50 & -0.498646 & $-0.1540(-2)$ & 0.55 & -0.48902 & $-0.9885(-2)$ \\
\hline 0.495 & -0.498630 & $-0.1599(-2)$ & 0.50 & -0.48703 & $-0.1391(-1)$ \\
\hline \multirow[t]{2}{*}{0.490} & -0.498620 & $-0.1666(-2)$ & 0.495 & -0.48643 & $-0.1463(-1)$ \\
\hline & & & 0.490 & -0.48557 & $-0.1588(-1)$ \\
\hline
\end{tabular}

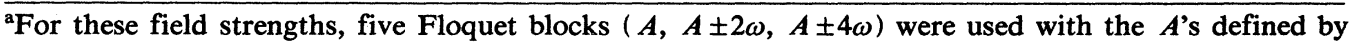
use of $15 s, 15 p, 15 d, 15 f, 15 g$ atomic $L^{2}$ functions with $\lambda=1.2$ and $\alpha=0.45 \mathrm{rad}$.

b $-0.1253(-3) \equiv-0.1253 \times 10^{-3}$.

'Six Floquet blocks $(A, A \pm 2 \omega, A \pm 4 \omega, A-6 \omega$ ) were used with each containing $20 s, 20 p, 20 d, 20 f$, $20 \mathrm{~g}, 20 \mathrm{~h}$ atomic $L^{2}$ functions with $\lambda=1.2$ and $\alpha=0.45 \mathrm{rad}$. 


\section{MULTIPHOTON IONIZATION AND THRESHOLD SHIFT}

The complex quasienergies $\left(E_{R},-\Gamma / 2\right) \equiv E_{R}-i \Gamma / 2$ of the perturbed ground state of atomic hydrogen obtained from the $L^{2}$ non-Hermitian Floquet calculations (converged to the digits shown) are listed in Tables I and II for several medium to high laser field strengths. Table I shows the results for laser frequencies nearby the onephoton-dominant ionization $\left(N_{m}=1\right)$ region $(\omega \sim 0.5$ a.u. $)$, while Table II for frequencies nearby the two-photondominant ionization $\left(N_{m}=2, \omega \geq 0.25\right.$ a.u. $)$ and threephoton-dominant ionization ( $N_{m}=3, \omega \sim 0.2$ a.u.) regions. $E_{R}$ 's are the ac Stark-shifted energies which determine the MPI thresholds (ionization potentials), shown below, whereas $\Gamma$ 's amount to the total MPI widths (rates).

To determine the MPI thresholds, we first look at the motion of a free electron in a linearly polarized field. Using the results of Szöke ${ }^{11}$ or the recent analysis of Cook et al. ${ }^{19}$ one finds that the electronic motion in a highfrequency time-dependent monochromatic field is governed by a Schrödinger equation with timeindependent effective potential $V_{\text {eff }}=e^{2} F^{2} / 4 m \omega^{2}$. Assuming the electric field (peak) amplitude $F$ is independent of the distance $r$, one sees that the electron energy in a linearly polarized wave is

$$
\epsilon_{\mathrm{el}}(F)=V_{\mathrm{eff}}+\epsilon_{T}=\frac{1}{4} \frac{e^{2} F^{2}}{m \omega^{2}}+\frac{P_{T}^{2}}{2 m},
$$

where $\epsilon_{T}$ is the kinetic energy of the electron. The effective potential $V_{\text {eff }}$ is known as the pondermotive potential which can also be regarded as the average oscillating energy of the electron in the field $\bar{\epsilon}_{\text {osc }}$. The latter is very small

TABLE II. Intensity-dependent complex quasienergy $\left(E_{R},-i \Gamma / 2\right)$ in atomic units of the perturbed ground state of the $\mathbf{H}$ atom nearby the $N_{m}=2\left(\omega \geq 0.25\right.$ a.u.) and $N_{m}=3(\omega \sim 0.2$ a.u.) regions.

\begin{tabular}{|c|c|c|c|c|c|}
\hline$\omega$ & $E_{R}$ & $-\Gamma / 2$ & $\omega$ & $E_{R}$ & $-\Gamma / 2$ \\
\hline \multicolumn{3}{|c|}{$F_{\mathrm{rms}}=0.01^{\mathrm{a}}$ a.u. } & \multicolumn{3}{|c|}{$F_{\mathrm{rms}}=-0.075^{\mathrm{c}}$ a.u. } \\
\hline 0.30 & -0.5005167 & $-0.3769(-5)$ & 0.30 & -0.51301 & $-0.639(-2)$ \\
\hline 0.28 & -0.5004353 & $-0.4513(-5)$ & 0.28 & -0.51215 & $-0.815(-2)$ \\
\hline 0.27 & -0.5004059 & $-0.5021(-5)$ & 0.27 & -0.51203 & $-0.920(-2)$ \\
\hline 0.26 & -0.5003816 & $-0.5616(-5)$ & 0.26 & -0.51243 & $-0.110(-1)$ \\
\hline 0.25 & -0.5003613 & $-0.6286(-5)$ & 0.22 & -0.51944 & $-0.173(-1)$ \\
\hline 0.22 & -0.5003353 & $-0.1799(-6)$ & 0.20 & -0.51892 & $-0.683(-2)$ \\
\hline 0.20 & -0.5002932 & $-0.1055(-6)$ & 0.19 & -0.51733 & $-0.437(-2)$ \\
\hline 0.19 & -0.5002452 & $-0.7147(-5)$ & 0.18 & -0.51484 & $-0.376(-2)$ \\
\hline \multirow[t]{2}{*}{0.18} & -0.5002898 & $-0.7911(-6)$ & & & \\
\hline & \multicolumn{2}{|c|}{$F_{\mathrm{rms}}=0.025^{\mathrm{a}}$ a.u. } & & \multicolumn{2}{|c|}{$F_{\mathrm{rms}}=0.10^{c}$ a.u. } \\
\hline 0.30 & -0.502899 & $-0.1314(-3)$ & 0.30 & -0.5158 & $-0.167(-1)$ \\
\hline 0.28 & -0.502474 & $-0.1608(-3)$ & 0.28 & -0.5178 & $-0.228(-1)$ \\
\hline 0.27 & -0.502320 & $-0.1800(-3)$ & 0.27 & -0.5250 & $-0.252(-1)$ \\
\hline 0.26 & -0.502197 & $-0.2021(-3)$ & 0.26 & -0.5315 & $-0.103(-1)$ \\
\hline 0.25 & -0.502100 & $-0.2259(-3)$ & 0.22 & -0.5355 & $-0.163(-1)$ \\
\hline 0.22 & -0.502192 & $-0.1888(-4)$ & 0.20 & -0.5250 & $-0.821(-2)$ \\
\hline 0.20 & -0.501585 & $-0.4667(-4)$ & 0.19 & -0.5208 & $-0.961(-2)$ \\
\hline 0.19 & -0.502396 & $-0.4458(-3)$ & 0.18 & -0.5188 & $-0.132(-1)$ \\
\hline 0.18 & -0.501968 & $-0.9484(-4)$ & & & \\
\hline \multicolumn{6}{|c|}{$F_{\mathrm{rms}}=0.05^{\mathrm{b}}$ a.u. } \\
\hline 0.30 & -0.50850 & $-0.1610(-2)$ & & & \\
\hline 0.28 & -0.50748 & $-0.2040(-2)$ & & & \\
\hline 0.27 & -0.50716 & $-0.2313(-2)$ & & & \\
\hline 0.26 & -0.50701 & $-0.2606(-2)$ & & & \\
\hline 0.25 & -0.50694 & $-0.2761(-2)$ & & & \\
\hline 0.22 & -0.50682 & $-0.5113(-3)$ & & & \\
\hline 0.20 & -0.50891 & $-0.3495(-2)$ & & & \\
\hline 0.19 & -0.50845 & $-0.2137(-2)$ & & & \\
\hline 0.18 & -0.50816 & $-0.1484(-2)$ & & & \\
\hline
\end{tabular}

${ }^{a}$ Five Floquet blocks $(A, A \pm 2 \omega, A \pm 4 \omega), 15 s, 15 p, 15 d, 15 f, 15 g L^{2}$ basis, $\lambda=1.2, \alpha=0.45$ rad. bSix Floquet blocks $(A, A \pm 2 \omega, A \pm 4 \omega, A-6 \omega), 15 s, 15 p, 15 d, 15 f, 15 g, 15 h$ basis, $\lambda=1.2, \alpha=0.45$ rad.

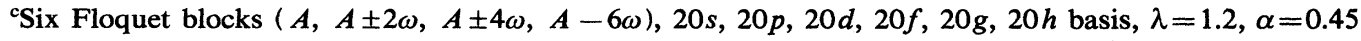
rad. 
for atomic hydrogen $\left(N_{m} \leq 3\right)$ at low field strengths. But for many-photon ionization, i.e., small $\omega$, as in the experiments of Refs. 1-3, this can be quite large. This highfrequency approximation will be valid provided $V_{\text {eff }} \epsilon_{T} \lesssim(\hbar \omega)^{2}$. The pondermotive potential acts to expel the electron from the laser. Thus an electron with energy $\epsilon_{\mathrm{el}}(F)$ less than $\bar{\epsilon}_{\text {osc }}$ cannot escape from the Coulomb potential and is trapped. Since in the limit of high quantum numbers a Rydberg electron becomes a free electron, the continuum threshold is shifted $\mathrm{up}^{20}$ by the amount equal to $\bar{\epsilon}_{\text {osc }}$. The ionization potential in intense fields can therefore be defined as

$$
\epsilon_{\mathrm{th}}(F)=\left|\frac{1}{4} \frac{e^{2} F^{2}}{m \omega^{2}}-E_{R}(F)\right|
$$

and the threshold shift as

$$
\Delta \epsilon_{\mathrm{th}}(F)=\epsilon_{\mathrm{th}}(F)-\epsilon_{\mathrm{th}}(F=0),
$$

where $E_{R}(F)$ is the field-dependent perturbed groundstate energy listed in Tables I and II and $\epsilon_{\mathrm{th}}(F=0)=0.5$ a.u. The total energy of the emitted electron in the field is then

$$
\epsilon_{\mathrm{el}}(F)=N \hbar \omega+E_{R}(F)=\frac{1}{4} \frac{e^{2} F^{2}}{m \omega^{2}}+\frac{P_{T}^{2}}{2 m},
$$

where $N\left(=N_{m}+S\right)$ is the total number of photons absorbed while the electron is near the atom. Since a free electron cannot absorb or emit photons once outside the Coulomb field, the electron has an energy $\epsilon_{\mathrm{el}}$ which is the same in the laser field and at the detector. The pondermotive potential plays an important role in altering the kinetic energy $\left(P_{T}^{2} / 2 m\right)$ of the electron from its value outside the laser field to a lower value inside the laser. We also note that, in actuality, the ground-state energy is uncertain by an amount $\Gamma$, so the actual threshold must be smeared (convolved) by this amount. This smearing will be small for results reported here, since the ac Stark shift $\Delta E_{R}$ (see below) is large compared to $\Gamma$ for all calculated values.

Table III shows typical examples of threshold shift calculations corresponding to $\omega=0.5$ a.u. $\left(N_{m}=1\right)$, 0.28 a.u. $\left(N_{m}=2\right)$, and 0.2 a.u. $\left(N_{m}=3\right)$, where $\Delta E_{R}$ $=E_{R}(F)-E_{R}(F=0)$ is the ac Stark shift, $\Delta E_{c}=e^{2} F^{2} / 4 m \omega^{2}$ the continuum threshold upshift, and $\Delta \epsilon_{\mathrm{th}}(F)$ the net threshold shift. Notice the marked difference in $\Delta \epsilon_{\text {th }}$ for $N_{m}=1$ and $N_{m} \geq 2$ cases. In the $N_{m}=1$ case, both the ground state and the continua are shifted upward with the former shift being larger. The result is that $\Delta \epsilon_{\text {th }}$ becomes more negative as $F$ increases, indicating the ionization potential decreases with increasing field strength $F$. For $N_{m} \geq 2$, the ac Stark shift of the ground state is downward while the continua are shifted up, yielding a larger net threshold shift, and the ionization potential increases nonlinearly with increasing field strength.
TABLE III. Intensity-dependent threshold shift $\Delta \epsilon_{\text {th }}=\Delta E_{c}-\Delta E_{R}$ for $\omega=0.5$ a.u. $\left(N_{m}=1\right), 0.28$ a.u. $\left(N_{m}=2\right)$, and 0.20 a.u. $\left(N_{m}=3\right) . \Delta E_{R}=E_{R}(F)-E_{R}(F=0)$ is the ac Stark shift of the ground state, while $\Delta E_{c}=e^{2} F^{2} / 4 m \omega^{2}$ $=e^{2} F_{\mathrm{rms}}^{2} / 2 m \omega^{2}$ is the continuum threshold upshift.

\begin{tabular}{llccc}
\hline \hline $\begin{array}{c}\omega \\
\text { (a.u.) }\end{array}$ & $\begin{array}{l}F_{\text {rms }} \\
\text { (a.u.) }\end{array}$ & $\begin{array}{c}\Delta E_{R} \\
(\mathrm{eV})\end{array}$ & $\begin{array}{c}\Delta E_{c} \\
(\mathrm{eV})\end{array}$ & $\begin{array}{c}\Delta \epsilon_{\text {th }} \\
(\mathrm{eV})\end{array}$ \\
\hline 0.5 & 0.01 & $5.870(-3)$ & $5.442(-3)$ & $-4.28(-4)$ \\
& 0.025 & $3.685(-2)$ & $3.402(-2)$ & $-2.83(-3)$ \\
& 0.05 & $1.485(-1)$ & $1.361(-1)$ & $-1.24(-2)$ \\
& 0.075 & $3.529(-1)$ & $3.061(-1)$ & $-4.68(-2)$ \\
0.28 & 0.01 & $-1.185(-2)$ & $1.735(-2)$ & $2.92(-2)$ \\
& 0.025 & $-6.732(-2)$ & $1.085(-1)$ & $1.76(-1)$ \\
& 0.05 & $-2.036(-1)$ & $4.339(-1)$ & $6.38(-1)$ \\
& 0.075 & $-3.306(-1)$ & $9.762(-1)$ & 1.307 \\
0.20 & 0.01 & $-7.979(-3)$ & $3.402(-2)$ & $4.20(-2)$ \\
& 0.025 & $-4.313(-2)$ & $2.126(-1)$ & $2.56(-1)$ \\
& 0.05 & $-2.425(-1)$ & $8.504(-1)$ & 1.093 \\
& 0.075 & $-5.149(-1)$ & 1.9133 & 2.428 \\
\hline \hline
\end{tabular}

Furthermore, the $\Delta E_{c}$ term becomes more and more important compared to $\Delta E_{R}$ as $N_{m}$ increases or $\omega$ decreases. The net result is that the ionization potential increases rapidly with both $F$ and $N_{m}$. The disappearance of the lowest energy electrons in the xenon MPI-ATI experiment of Kruit et al. ${ }^{2}\left(N_{m}=11\right)$ was thought to be merely a consequence of this fact. One should also not be surprised to see the significant effect of the ground-state ac Stark shift $\Delta E_{R}$ in determining the threshold for MPI of atomic hydrogen, particularly for small $N_{m}$ or large $\omega$. The hydrogen atom is much more polarizable than, for example, rare-gas atoms.

\section{ABOVE-THRESHOLD \\ IONIZATION-BRANCHING-RATIO ESTIMATION}

Besides the threshold shift discussed above, another interesting feature of recent MPI experiments is the ATI phenomenon which involves the decay of a discrete state coupled to multiple continua. Perturbative calculations of the (weak-field) ATI rates for atomic hydrogen have been performed by several workers ${ }^{21}$ based on various techniques: Sturmian expansion, Padé approximant, and implicit summation techniques. Nonperturbative treatments of ATI in strong fields are available but all are based on simple model problems. ${ }^{4-10}$ The latter studies nevertheless provide useful qualitative insights regarding the mechanisms in ATI processes. In the following we present an $a b$ initio estimate of the branching ratios in the ATI of atomic hydrogen in intense fields.

The transition probability of going from the initial (unperturbed) Floquet state $|\alpha, 0\rangle$ to the final Floquet state $|\beta, N\rangle$, is

$$
P_{\alpha 0 \rightarrow \beta N}(t)=\left|\frac{1}{2 \pi i} \oint d z e^{-i z t}\left\langle\beta, N\left|\frac{1}{z-\hat{H}_{F}(\alpha)}\right| \alpha, 0\right\rangle\right|^{2}
$$


where $\hat{H}_{F}(\alpha)$ is the dilation-transformed Floquet Hamiltonian, with

$$
\hat{H}_{F}(\alpha)\left|\lambda_{\gamma l}\right\rangle=\lambda_{\gamma l}\left|\lambda_{\gamma l}\right\rangle \text {, }
$$

where $\lambda_{\gamma l}$ and $\left|\lambda_{\gamma l}\right\rangle$ are, respectively, the (perturbed) complex quasienergy eigenvalues and eigenvectors, and $\lambda_{\gamma l}=E_{\gamma l}-i \Gamma_{\gamma} / 2$ and $E_{\gamma l}=E_{\gamma}+l \hbar \omega$. Equation (6) can be rewritten as

$$
\begin{aligned}
P_{\alpha 0 \rightarrow \beta N}(t) & =\left|\frac{1}{2 \pi i} \sum_{\gamma, l} \oint d z e^{-i z t}\left\langle\beta, N \mid \lambda_{\gamma l}\right\rangle \frac{1}{z-\lambda_{\gamma l}}\left\langle\lambda_{\gamma l} \mid \alpha, 0\right\rangle\right|^{2} \\
& =\left|\sum_{\gamma, l}\left\langle\beta, N \mid \lambda_{\gamma l}\right\rangle\left\langle\lambda_{\gamma l} \mid \alpha, 0\right\rangle e^{-i \lambda_{\gamma l} t^{t}}\right|^{2},
\end{aligned}
$$

which shows that all complex QES $\left|\lambda_{\gamma l}\right\rangle$ of Eq. (7) are required to obtain the transition probability. Furthermore, in the original $L^{2}$ non-Hermitian Floquet approach, ${ }^{13}|\alpha\rangle$ and $|\beta\rangle$ are dilated atomic states simulated by $L^{2}$ functions and, in particular, positive energy $|\beta\rangle$ states are not genuine continuum states but pseudocontinuum states. Thus within the $L^{2}$ complex coordinate approach, it is difficult, in general, to achieve convergence of the branching ratios to an individual continuum. ${ }^{22}$ This problem can be partially overcome, however, by noting that the initial $(|\alpha, 0\rangle)$ and the final $(|\beta, N\rangle)$ Floquet states can actually be chosen at will and need not be the eigenfunctions of $\hat{H}_{F}(\alpha)$. In other words, Eqs. (6) and (8) are still valid if we replace $|\alpha\rangle$ and $|\beta\rangle$ by the exact (un- dilated) bound and continuum atomic states. Thus $\left\langle\beta, N \mid \lambda_{\gamma l}\right\rangle$ will now be interpreted as the projection of the QES $\left|\lambda_{\gamma l}\right\rangle$ onto the Floquet state $|\beta, N\rangle \equiv|\beta\rangle_{\otimes}|N\rangle$, where $|\beta\rangle$ is the physical (undilated) atomic continuum state. Furthermore, the sum over $\gamma, l$ in Eq. (8) may be avoided in some long-time limit. Since the imaginary widths $\Gamma_{\gamma}$ of excited bound states and pseudocontinuum states are usually larger to much larger than the ground-state width $\Gamma_{1 S}$ (since decay from ground state represents the slowest process), there exists a longtime regime where all states (with complex energies $\lambda_{\gamma l}$ ) have decayed away but $\left|\lambda_{1 S, 0}\right\rangle$. In that case, Eq. (8) can be approximated by

$$
P_{1 S, 0 \rightarrow \epsilon_{l},-N}(t) \simeq\left|\left\langle\epsilon_{l},-N \mid \lambda_{1 S, 0}\right\rangle\right|^{2}\left|\left\langle\lambda_{1 S, 0} \mid 1 S, 0\right\rangle\right|^{2} e^{-\Gamma_{1 S} t},
$$

and the branching ratio to an individual continuum approximated by

$$
\frac{P_{1 S, 0 \rightarrow \epsilon_{l_{1}},-N_{1}}(t)}{P_{1 S, 0 \rightarrow \epsilon_{l_{2}}, N_{2}(t)}} \simeq \frac{\left|\left\langle\epsilon_{l_{1}},-N_{1} \mid \lambda_{1 S, 0}\right\rangle\right|^{2}}{\left|\left\langle\epsilon_{l_{2}},-N_{2} \mid \lambda_{1 S, 0}\right\rangle\right|^{2}},
$$

where $\left|\epsilon_{l},-N\right\rangle$ can be regarded as the Floquet state with atomic continuum energy $\epsilon_{l}$ and partial wave $l$ which can be achieved by absorption of $N$ photons from the unperturbed ground state $|1 S, 0\rangle$. Equation (10) requires the information of only one QES eigenvector ${ }^{23}\left|\lambda_{1 S, 0}\right\rangle$, which is expressed in terms of $L^{2}$ Laguerre functions, and the calculation of overlap matrix elements between the undilated atomic continuum states $\left|\epsilon_{l}\right\rangle$ and $L^{2}$ Laguerre basis. $\left|\epsilon_{l}\right\rangle$ 's are determined by the solution of the Schrödinger equation for the motion of a free electron subjected to the Coulomb and the (constant for a given $F$ ) pondermotive potentials, with the total energy given by (2), i.e., they are Coulomb wave functions with kinetic energy corresponding to $\epsilon_{\mathrm{el}}-\bar{\epsilon}_{\mathrm{osc}}$.

Table IV shows some representative branching ratio estimates for multiphoton absorption above the ionization threshold by atomic hydrogen for the cases of $N_{m}=1,2$, and 3, and $N=1$ to 6 , where $N_{m}$ is the minimum number of photons needed to ionize the atom while $N$ is the total number of photons absorbed. The energies and fields are such that the use of the pondermotive potential will be well valid for all except the highest electron energies and fields [where it is only marginally valid (i.e., $\left.\left.e^{2} F^{2} \epsilon_{T} / 4 m \hbar^{2} \omega^{4} \sim 1\right)\right]$. The frequency and intensity dependence of the ATI processes were found to be not very sensitive to details of calculations. The general trend is well illustrated in Table IV and can be summarized as follows: (a) At small $N_{m}$, only the lowest one- or twocontinuum peaks (i.e., $N=N_{m}, N_{m}+1$ ) are expected to be visible. This persists to even rather high intensities. However, as $N_{m}$ becomes larger (or $\omega$ becomes smaller), there is a drift to more continua and more peaks can be observed, but the first two peaks nearest the threshold are always the first ones to get saturated. The saturation intensity for continuum-continuum transitions is seen to decrease rather rapidly with decreasing $\omega$. (b) At a given frequency, the first continuum peak is always the highest to start with. When the laser intensity increases, the first peak decreases, and the second peak begins to increase and eventually becomes the highest. It is clear that the flux moves to higher continua as intensity increases; see in particular the case $\omega=0.2$ a.u. It is also interesting to note that the first peak could become small enough before the ionization potential becomes large enough to prevent its appearance. Thus the disappearance of the first peak seen in the experiments ${ }^{2}$ can be attributed to the effect due to either the drift of the flux to higher continua or the in- 
TABLE IV. Branching ratio estimation for multiphoton absorption above the ionization threshold by the $\mathrm{H}$ atom. $P(N)$ is the relative population of the continuum corresponding to absorption of $N$ photons from the ground state. $N_{m}$ is the minimum number of photons required to ionize the atoms.

\begin{tabular}{ccccccccc}
\hline \hline $\begin{array}{c}\omega \\
\text { (a.u.) }\end{array}$ & $N_{m}$ & $\begin{array}{c}F_{\text {rms }} \\
\text { (a.u.) }\end{array}$ & $P(1)$ & $P(2)$ & $P(3)$ & $P(4)$ & $P(5)$ & $P(6)$ \\
\hline 0.50 & 1 & 0.010 & 1.0 & $5.6(-4)$ & & & \\
& 1 & 0.025 & 1.0 & $5.8(-3)$ & $4.7(-5)$ & & \\
& 1 & 0.050 & 1.0 & 0.012 & $2.7(-4)$ & & \\
& 1 & 0.075 & 1.0 & 0.015 & $8.0(-4)$ & & & \\
0.28 & 2 & 0.010 & & 1.0 & $1.6(-4)$ & & & \\
& 2 & 0.025 & & 1.0 & $1.0(-3)$ & $6.5(-6)$ & & \\
& 2 & 0.065 & & 1.0 & 0.01 & $1.8(-4)$ & $4.4(-6)$ & \\
& 2 & 0.075 & & 1.0 & 0.05 & $8.8(-4)$ & $2.7(-5)$ & \\
& 2 & 0.080 & & 1.0 & 0.14 & $3.0(-3)$ & $8.4(-5)$ & \\
& 2 & 0.083 & & 1.0 & 0.26 & $6.2(-3)$ & $3.7(-4)$ & \\
& 2 & 0.085 & & 1.0 & 1.27 & $2.7(-2)$ & $1.9(-3)$ & \\
& 3 & 0.100 & & $\mathrm{a}$ & 1.00 & 0.03 & $8.3(-4)$ & $9.1(-6)$ \\
& & & & & & & & \\
0.20 & 3 & 0.010 & & & 1.0 & $3.0(-4)$ & & \\
& 3 & 0.025 & & & 1.0 & $1.5(-3)$ & $8.1(-6)$ & \\
& 3 & 0.050 & & & 1.0 & 0.08 & $1.6(-3)$ & $3.2(-5)$ \\
& 3 & 0.060 & & & 1.0 & 0.23 & $1.3(-2)$ & $3.6(-4)$ \\
& 3 & 0.070 & & & 1.0 & 0.58 & $6.4(-2)$ & $2.6(-3)$ \\
& 3 & 0.075 & & & 1.0 & 1.82 & 0.26 & 0.01 \\
& 3 & 0.078 & & & 1.0 & 5.53 & 0.88 & 0.05 \\
& 4 & 0.080 & & & $\mathrm{a}$ & 1.0 & 0.20 & 0.06 \\
\hline \hline
\end{tabular}

${ }^{a}$ Electron peak disappears due to the threshold increases.

crease of the ionization potential, or both. There is, however, no firm evidence in our present calculations that subsequent peaks are "switched."

In conclusion, we have presented in this paper accurate results for intensity dependent threshold shift and ionization potentials of atomic hydrogen in the presence of strong laser fields. The data show the importance of the ac Stark shift and the pondermotive potential in the determination of the net threshold shift. Our estimate of the branching ratios to individual continuum also leads to useful insights regarding the general feature and mechanisms in the above-threshold ionization processes.

\section{ACKNOWLEDGMENTS}

One of the authors (S.I.C.) acknowledges support from the Department of Energy (Division of Chemical Sciences), from the Donors of the Petroleum Research Fund, administered by the American Chemical Society, and from the JILA Visiting Fellow Program. J.C. acknowledges support from the National Science Foundation Grant PHY82-00805 through the University of Colorado and from the Atomic and Plasma Radiation Division of NBS, and stimulating and informative conversations with D. E. Kelleher and A. Szöke.
*Permanent address: Department of Chemistry, University of Kansas, Lawrence, Kansas 66045.

${ }^{1}$ F. Fabre, G. Petite, P. Agostini, and M. Clement, J. Phys. B 15, 1353 (1982); P. Agostini, F. Fabre, G. Mainfray, and N. K. Rahman, Phys. Rev. Lett. 42, 1127 (1979).

${ }^{2}$ P. Kruit, J. Kimman, H. G. Muller, and M. J. van der Wiel, Phys. Rev. A 28, 248 (1983).

${ }^{3}$ U. Johann, T. S. Luk, H. Egger, H. Plummer, and C. K. Rhodes (unpublished).

${ }^{4}$ H. G. Muller, A. Tip, and M. J. van der Wiel, J. Phys. B 16, L679 (1983); H. G. Muller and A. Tip, Phys. Rev. A 30, 3039 (1984).

${ }^{5}$ M. H. Mittleman, Phys. Rev. A 29, 2245 (1984); J. Phys. B 17, L351 (1984).
6Z. Białynicka-Birula, J. Phys. B 17, 2091 (1984).

${ }^{7}$ M. Edwards, L. Pan, and L. Armstrong, Jr., J. Phys. B 17, L515 (1984).

${ }^{8}$ Z. Deng and J. H. Eberly, Phys. Rev. Lett. 53, 1810 (1984); J. Opt. Soc. Am. B 2, 486 (1985).

${ }^{9}$ K. Rzażewski and R. Grobe, Phys. Rev. Lett. 54, 1729 (1985); and (unpublished).

10M. Crance and M. Aymar, J. Phys. B 13, L421 (1980).

${ }^{11}$ A. Szöke, J. Phys. B 18, L427 (1985).

${ }^{12}$ D. E. Kelleher, M. Ligare, and L. R. Brewer, Phys. Rev. A 31, 2747 (1985), and references therein.

${ }^{13}$ S.-I Chu and W. P. Reinhardt, Phys. Rev. Lett. 39, 1195 (1977); A. Maquet, S.-I Chu, and W. P. Reinhardt, Phys. Rev. A 27, 2946 (1983). 
14J. H. Shirley, Phys. Rev. 138, B979 (1965).

${ }^{15}$ Reviews of the use of complex coordinates (dilation analyticity) in atomic and molecular physics can be found in: W. P. Reinhardt, Annu. Rev. Phys. Chem. 33, 223 (1982); B. R. Junker, Adv. At. Mol. Phys. 18, 207 (1982).

${ }^{16}$ S.-I Chu, J. Chem. Phys. 75, 2215 (1981); S.-I Chu, C. Laughlin, and K. K. Datta, Chem. Phys. Lett. 98, 476 (1983).

${ }^{17}$ S.-I Chu, Chem. Phys. Lett. 54, 367 (1978).

${ }^{18}$ H. A. Yamani and W. P. Reinhardt, Phys. Rev. A 11, 1144 (1975).

${ }^{19}$ R. J. Cook, D. G. Shankland, and A. L. Wells, Phys. Rev. A 31, 564 (1985).

20P. Avan, C. Cohen-Tannoudji, J. Dupont-Roc, and C. Fabre, J. Phys. (Paris) 37, 993 (1976); S. Liberman, J. Pinard, and A. Taleb, Phys. Rev. Lett. 50, 888 (1983); L. Hollberg and J. L.
Hall, ibid. 53, 230 (1984).

${ }^{21}$ Y. Gontier and M. Trahin, J. Phys. B 13, 4383 (1980), and references therein; M. Aymar and M. Crance, ibid. 14, 3585 (1981).

${ }^{22}$ This is true particularly for long-range (such as Coulomb) potentials. In fact, the dilated Coulomb continuum wave functions diverge exponentially at large $r$. For a recent discussion on the feasibility of using the $L^{2}$ complex coordinate technique in the calculation of bound-free amplitudes in the presence of somewhat shorter range potentials (such as $-1 / r^{4}$ ), see B. R. Johnson and W. P. Reinhardt, Phys. Rev. A 28, 1930 (1983).

${ }^{23}$ Inverse iteration technique was used to obtain only one eigenvalue and eigenvector of $\hat{H}_{F}(\alpha)$. 